\title{
PRODUCING AND VISUALIZING A COUNTRY-WIDE 3D DATA REPOSITORY IN FINLAND
}

\author{
H. Visuri ${ }^{1, *}$, J. Jokela $^{2}$, N. Mesterton ${ }^{3}$, P. Latvala ${ }^{1}$, T. Aarnio ${ }^{1}$ \\ ${ }^{1}$ National Land Survey of Finland, Department of SDI Services - (hanna.visuri, pekka.latvala, timo.aarnio)@nls.fi \\ ${ }^{2}$ National Land Survey of Finland, Department of Topographic Data - joonas.jokela@nls.fi \\ ${ }^{3}$ National Land Survey of Finland, Department of Application Services- nils.mesterton@nls.fi
}

KEY WORDS: 3D SDI, 3D Data Production, 3D Visualization, Spatial Data Harmonization, Spatial Data Quality

\begin{abstract}
:
The amount and the quality of 3D spatial data are growing constantly, but the data is collected and stored in a distributed fashion by various data collecting organizations. This may lead to problems regarding interoperability, usability and availability of the data. Traditionally, national spatial data infrastructures have focused on 2D data, but recently there has been great progress towards introducing also 3D spatial data in governmental services. This paper studies the process of creating a country-wide 3D data repository in Finland and visualizing it for the public by using an open source map application. The 3D spatial data is collected and stored into one national topographic database that provides information for the whole society. The data quality control process is executed with an automated data quality module as a part of the import process to the database. The 3D spatial data is served from the database for the visualization via 3D service and the visualization is piloted in the National Geoportal.
\end{abstract}

\section{INTRODUCTION}

The production volume of 3D spatial data is growing constantly due to the utilization of remote sensing technologies, such as LiDAR, that enable the 3D data to be produced with higher resolution and lower costs. Although the amount of $3 \mathrm{D}$ data is growing, the data might not reach the users. The 3D spatial data is collected and stored in a distributed fashion by the various data collecting organizations, which makes it difficult for the users to find the specific data for their needs.

At the same time, we are only beginning to recognize the use cases that could benefit from the use of 3D spatial data. According to the recent research (Acil Allen Consulting and CRC for Spatial Information, 2017), by integrating the 3D data models of built environment produced by private companies with models produced by the public sector and making those available for potential users, even $20 \%$ of planning and construction costs could be saved. A recent report published by the Finnish Ministry of Agriculture and Forestry (Finnish Ministry of Agriculture and Forestry, 2018) states that the importance of 3D spatial data in design, building and maintenance workflows will grow in the near future. It is estimated that just data model-based building permit process will save approximately one million euros in an actively developing city in Finland.

Visualizing data on a map is a very efficient way of understanding and communicating various phenomena. Versatile visualizations may help to detect whole new dependencies between datasets. Traditionally map applications have had an important role in utilizing spatial information. This role can be seen growing even stronger in the future when $2 \mathrm{D}$ datasets are reinforced with 3D data information. As users have become accustomed to quality map services and applications for the use of 2D data, this sets high expectations for similar services for 3D data. Although the costs of 3D data and 3D applications have been reduced and technologies for generating and maintaining 3D information have matured, many governmental organizations are hesitating to introduce 3D applications and technologies in their processes. The hesitation in these organizations can be seen to be caused by the lack of knowledge in 3D domain and the shortcomings of the processes to handle the 3D information in the current Spatial Data Infrastructure (SDI). (Stoter et al., 2011)

National Spatial Data Infrastructures have generally focused on $2 \mathrm{D}$ data. Only recently there has been great progress in introducing 3D data in governmental services. Examples of 3D SDI can be found for example in Netherlands (Stoter et al., 2011) and UK (Capstick et al., 2007).

The report on spatial data policy in Finland (Finnish Ministry of Agriculture and Forestry, 2018) states that the future needs of transport infrastructure require precise and rich content of $3 \mathrm{D}$ information covering the entire country. The National Land Survey of Finland (NLS) will be responsible for the production of country-wide 3D data. The 3D data produced by the NLS will be enriched with the more accurate and detailed models produced by cities. This will create the foundation for the 3D SDI being developed. The harmonized and maintained 3D spatial dataset opens possibilities for developers and suppliers to innovate new products and services.

This paper studies the process of creating a country-wide 3D data repository and visualizing it for the public by using an open source map application. The structure of the paper is as follows. In section 2 we make an overview of the 3D SDI process. Section 3 describes in more detail the processing of the 3D data and section 4 the quality control of the 3D data. Finally, in section 5 we describe the process of visualizing the $3 \mathrm{D}$ data in a browserbased map application.

\section{OVERVIEW OF THE 3D SDI PROCESS}

Since 2017, the Public administrators in Finland have been working together to harmonize the spatial data of the state, municipalities and other regions and making them available for companies and communities. The work is implemented in the Geospatial Platform project. The goal of this project is to create a shared spatial data platform for the public administration. The spatial data platform will offer common specifications and services for the data producers in the public administration, 
shared and coherent data sets for all data users and common user services. The first phase of the project will be completed at the end of 2019.

The National Topographic Database program is a sub-program of the Geospatial Platform project. It digitalizes and unifies the core spatial data themes of Finland to one national topographic database (NTDB) that provides information for the whole society. The NTDB will include buildings and structures, traffic network, hydrography, land cover and elevation data. In addition, the database includes geographical names, aerial images, digital elevation models and laser scanning data. Data collection for the NTBD is performed by various municipalities and governmental agencies.

The data collection process where many organizations are producing the 3D data, may lead to inconsistent results. Therefore, a robust quality control process is needed. In the NTDB, the quality control process is executed with an automated data quality module called "QualityGuard", that works as a part of the import process to NTDB. Data producers can also use it independently to find possible errors in their data. QualityGuard returns a data quality report, which contains statistics and a quality error dataset regarding the imported data.

The data in the NTDB will be available for the public via different information services connected to the NTDB. 3D service will offer 3D buildings in the 3D Tiles format for the visualization. 3D Tiles is the Open Geospatial Consortium's (OGC) community standard (OGC, 2019).

The 3D data will be visualized in The National Geoportal of Finland. The National Geoportal is a browser-based map application developed and maintained in the National Land Survey of Finland and it includes over 1600 map layers from various data producers.

The National Geoportal is built on top of an open source map application platform called "Oskari". Oskari is developed originally in the NLS but nowadays there is a stakeholder community responsible for the development. Oskari is used widely by the Finnish authorities and other stakeholders to build their own browser-based map applications. By building 3D support to Oskari we enable visualization of 3D data not only in the National Geoportal but also in any map application built on top of Oskari. This works also as a driver for the data producers to import their 3D data to the NTDB.

Figure 1 shows the steps of the process from collecting the 3D data to visualizing it on a map application.

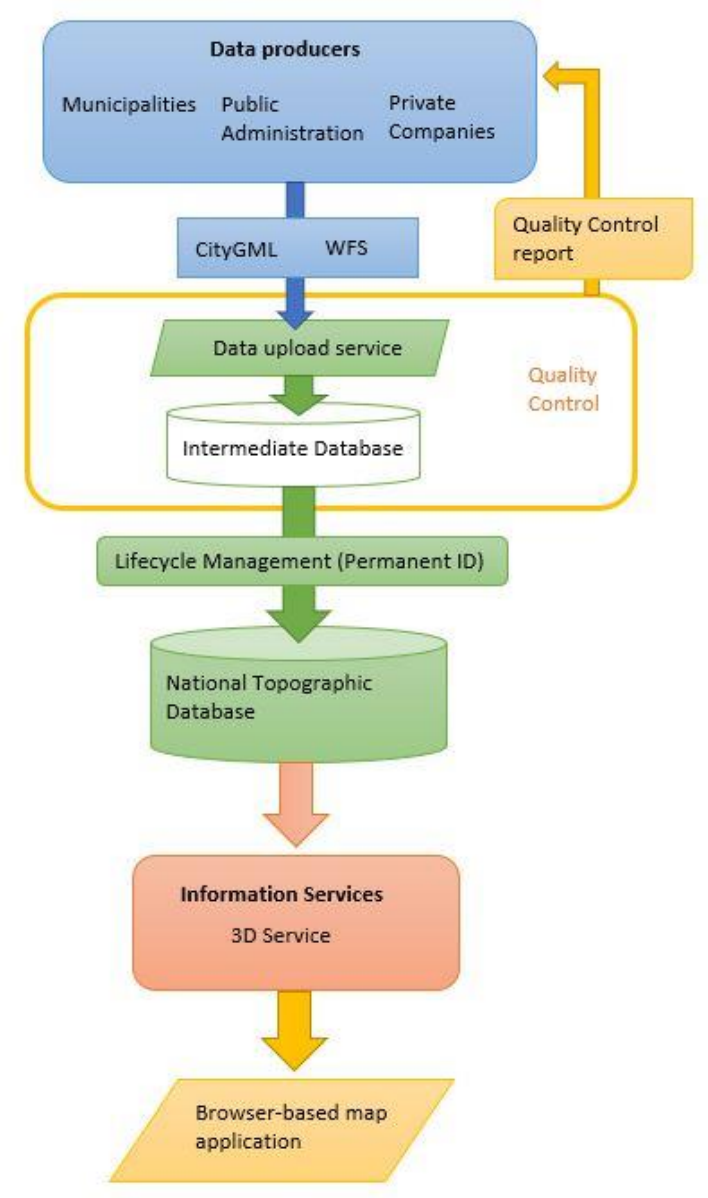

Figure 1 Geospatial Platform architecture

\section{3D DATA PRODUCTION AND MANAGEMENT}

$3 \mathrm{D}$ buildings were chosen as the starting point for the $3 \mathrm{D}$ development as several cities and other data producers already had existing workflows and datasets for them. This made it possible to test the early implementations with different $3 \mathrm{D}$ building products from different workflows.

As with 2D geospatial data, the 3D data collection for the NTDB is also performed by various organizations. The country-wide 3D data produced by the NLS will create the basis for the national $3 \mathrm{D}$ data repository. This data is supplemented by the, often higher quality, data produced by cities that produce their own 3D city models. The aim is to have the country-wide 3D data produced as consistent to CityGML LoD2. CityGML is an OGC community standard (OGC, 2012). The NTDB data model allows capture of the data in LoD0 to LoD3.

\subsection{D Building production}

Promotion of interoperability for the 3D models of different data producers is a vital step for the usability of 3D spatial data (Ahonen-Rainio et al., 2014). As the data is produced by municipalities and governmental agencies, interoperability is one of the major challenges. Currently there are no extensive national guidelines available in Finland for the modeling of 3D buildings. Typically, the 3D buildings are modelled either by using the OGC CityGML standard or the guidance provided by Special Interest Group 3D (SIG 3D, 2013). One of the problems with this 
approach is that the concept of LoDs is rather ambiguous leading to various interpretations of the 3D modelling as stated by Biljecki et al. (2014).

The national 3D modelling guidelines, as a part of the conceptual model, is currently being processed to be accepted as a national specification under the Public Administration Recommendations. This should be a common specification among the data producers and harmonize the spatial data in Finland, including the 3D. The specification is based on CityGML, INSPIRE, other Public Administration Recommendations, and similar guidelines from other countries. The specification follows the CityGML definitions but specifies them for the national needs.

The $2 \mathrm{D}$ and $3 \mathrm{D}$ data are maintained in the same database using the same processes and therefore they also share the same persistent IDs. These persistent IDs are given to each feature in the NTDB and remain static throughout each feature's lifecycle. With these IDs, the production and data management of 3D buildings can be based on existing $2 \mathrm{D}$ building data. The 3D geometries are part of the abstract building and cannot be imported into the database without an existing 2D geometry. When a 3D building is added to the NTDB, existing 2D geometries are checked against the 3D geometry to ensure that a correct corresponding 2D building is utilized in the NTDB. Since each feature corresponds to just one real world object and the different instances of the object's digital twins are linked to that feature, the data management process is simplified.

Since each feature in the NTDB is given a persistent ID, the NTDB also supports 4D data management. The changes to the feature's geometry, semantic or attribute data can be tracked through version numbering. This allows users to query and analyze data at different points in time.

The production of national 3D building data will be based on the new higher density laser scanning data alongside with the existing $2 \mathrm{D}$ building footprint data. The production is planned to be as automatic as possible with a human operator confirming that the quality of the produced data is within defined quality standards.

The produced 3D buildings will be modelled according to the modelling guidelines and conceptual model that are under development. As with CityGML version 2, the NTDB building can consist of one or more building parts. Although the division of a building into building parts is also rather ambiguous as mentioned in Eriksson et al. (2018) they also highlight the many benefits that can be achieved through building parts.

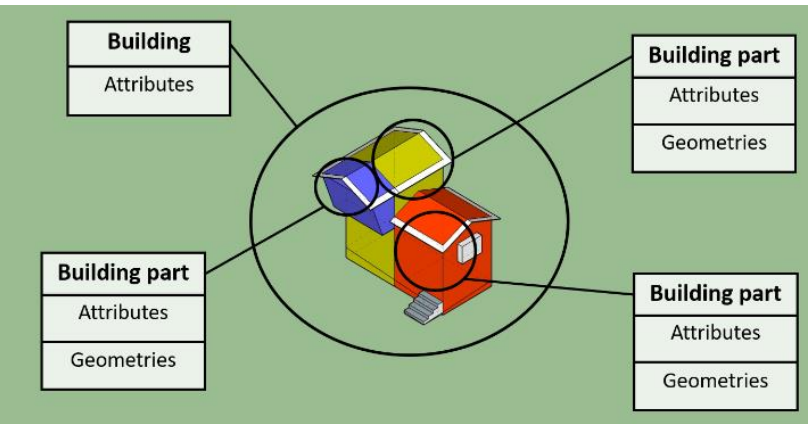

Figure 2 3D building model

\subsection{New Aerial Laser Scanning Program}

As stated in Oude Elberink et al. (2013) the increasing density of point cloud data has allowed the reconstruction of more detailed and reliable description of objects. Currently the program to laser-scan the whole country of Finland with the point density of $0.5 \mathrm{pts} / \mathrm{m}^{2}$ is almost finished. As the needs for higher point density data have arose in recent years, the NLS has started another laser scanning program to scan Finland with point density of $5 \mathrm{pts} / \mathrm{m}^{2}$. Each year approximately half of the scanning areas are flown during spring season without leaves on the trees and half during summer season with leaves on the trees. The different time windows can be seen to affect the quality of the 3D production as during the spring season there will be less occlusion from the trees. This highlights the importance of accurate metadata for the created products.

Among other benefits, the higher density point cloud data will allow the production of $3 \mathrm{D}$ buildings that are consistent with CityGML LoD2. The aerial laser scanning program is planned to cover the whole country every 6 years, excluding the most northern parts of Finland where the changes are seen to happen less frequently. Following this, also the $3 \mathrm{D}$ building production will cover and update the whole country in 6-year intervals.

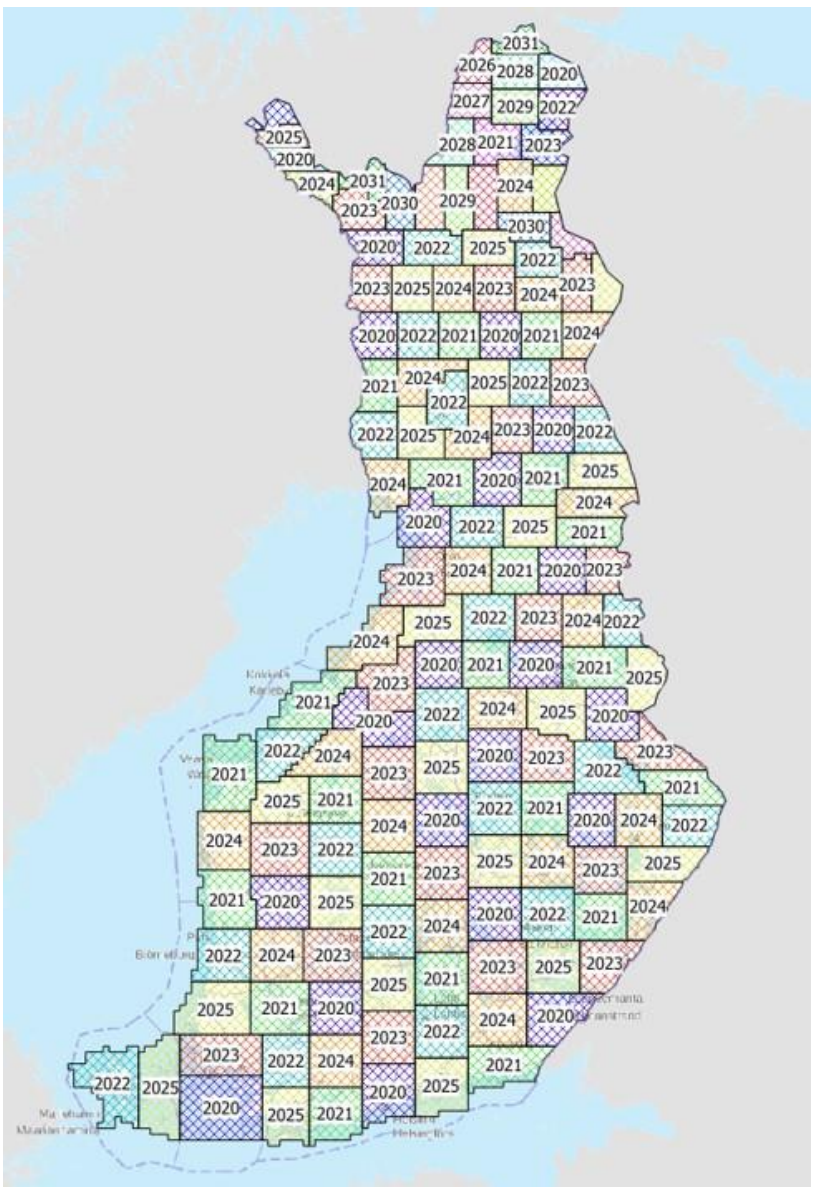

Figure 3. New laser scanning program production areas

\section{QUALITY CONTROL}

A key component in reaching better harmonization across the national spatial data in Finland will be an automated data quality evaluation process named as QualityGuard, which will be applied 
on all spatial data that will be imported to the national topographic database. QualityGuard works in two modes: a) as a part of the import process to NTDB, and b) independently. Users can validate their data using the QualityGuard to find possible errors in their data and fix them. Once the data is corrected and passes the validation process, the data producers can import their data to the NTDB. The data providers will receive a data quality report that contains statistics on the data quality and a quality error dataset regarding their imported data.

The data quality evaluation is based on validating the data's conformance against the data product specifications that are expressed as quality rules. Three different quality elements are utilized from the ISO 19157 standard: format consistency, domain consistency and topological consistency. The quality rules have been defined in co-operation with specialists in the field and the technical developing team. The quality rules concerning the geometrical consistency of 3D solids are derived mainly from the definition of solids described in ISO 19107: Geographic information - Spatial schema standard. The rules regarding the semantics are based on the logical order of faces and on the definitions in the CityGML standard.

A country-wide, centralized automated spatial data quality process is one of the key elements in order to harmonize the data that will be imported to the NTDB. Oude Elberink et al (2013) describe the many benefits that come with validated spatial data. One of these benefits is the interoperability between different systems and data formats, which is also one of the aims of the NTDB program.

The greater aim is to achieve compliance with the auditing process described in the ISO 19158 standard. The purpose of the quality control process is to ensure that the supplying organizations are capable of delivering data of expected quality. However, implementing a country-wide process is rather challenging because municipalities and other organizations might not have the capability or resources to repair the quality issues identified by the QualityGuard. The QualityGuard has enabled an automated spatial data quality validation process for incoming data and it is currently being evaluated in practice. The results have already been well received by the users. We believe this will be a common practice with all SDI datasets in Finland.

\section{DATA VISUALIZATION}

3D data from the NTDB will be visualized in the National Geoportal of Finland, which is built on top of Oskari. So far, Oskari has supported only 2D data, but during the project support for 3D buildings, point clouds and digital elevation model will be developed. Data visualization process consists of two parts: Map application for rendering the 3D data and 3D service for serving the 3D data for the application from the NTDB.

\subsection{D service}

In order to be able to show 3D data in a browser-based map application, the data must be in a format supported by the rendering $3 \mathrm{D}$ library. At the beginning of the project the available libraries for visualizing 3D data and formats for streaming 3D data were identified and two libraries were chosen for further analysis: Cesium JS and ArcGIS API for JS. Cesium JS and ArcGIS API for JS support different OGC community standards for streaming 3D data: Cesium JS supports 3D Tiles and ArcGIS API for JS supports I3S (OGC, 2017). Cesium JS is an open source library whereas ArcGIS API for JS is a commercial product, which means that Cesium JS was better option for this use case. Cesium JS is also possible to integrate easily to the Oskari Platform since it uses OpenLayers for mapping and there is available an open source library ol-Cesium to integrate CesiumJS 3D globe with the 2D map provided by OpenLayers.

Since Cesium JS was chosen for 3D map rendering it was natural to use 3D Tiles as a format for streaming the 3D data, but there aren't currently any open source tools for producing 3D Tiles from the 3D dataset. Cesium JS has developed Cesium ion platform for tiling and streaming 3D geospatial data, but it requires commercial licence for governmental use. In this project we used Cesium ion free community account for evaluating if Cesium ion would be suitable for our needs.

Cesium ion accepts CityGML or KML/Collada as input data, which means that the data from the NTDB must be first converted to CityGML. FME data integration platform was used for integrating database with Cesium ion since it enables transforming and transferring data from different formats to Cesium ion with CesiumIonConnector plugin. Cesium ion community account is hosted in the cloud and the produced $3 \mathrm{D}$ Tiles can be requested from there with specific id that is given to the tiles of each dataset. Figure 4 shows the architecture of the $3 \mathrm{D}$ service used in this project.

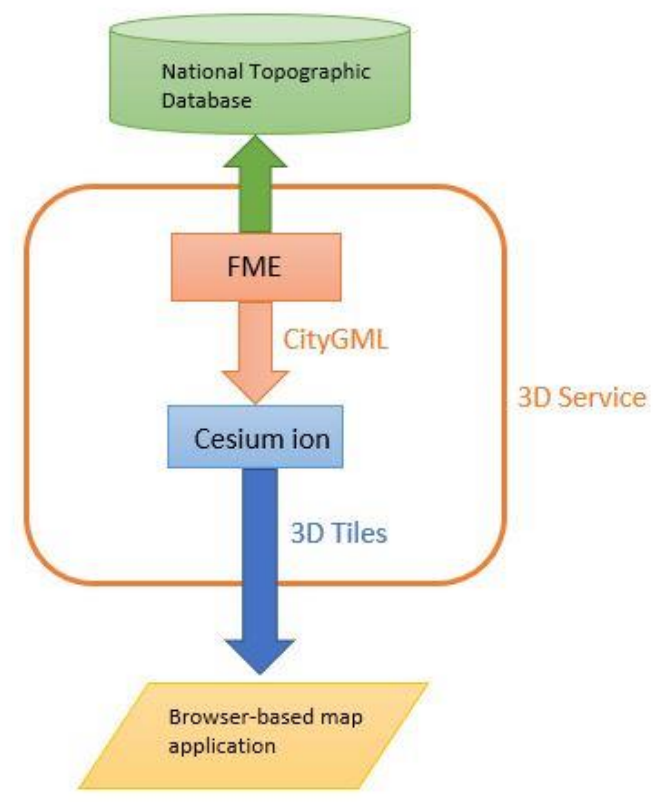

Figure 4. 3D Service components

\subsection{The National Geoportal and Oskari}

The National Geoportal of Finland has been for 10 years the platform for piloting the possibilities of geospatial data infrastructure. Since the National Land Survey of Finland is now producing a uniform $3 \mathrm{D}$ dataset from the whole country, there is a need to support also 3D data in the National Geoportal.

The National Geoportal is built on top of Oskari. Oskari can be used either as a standalone service or by publishing an embeddable map to be used on another website. The embedded map can be customized and interaction between the website and map can be added using the Oskari RPC API. This enables integrations to existing browser-based services and facilitates the 
development of new services which require a map component. The communication between the embedded map and the parent web page is two-way, i.e. user interactions can trigger changes in either one.

Since 3D support is built as part of Oskari, 3D features can be used in any map applications utilizing Oskari. Oskari enables reviewing the imported $3 \mathrm{D}$ data against any map layer available via standard interfaces such as Web Feature Service and Web Map Service. It will also enable displaying property information of buildings, rule-based rendering, time control and as the most important feature: offering embedded maps containing 3D spatial data.

\subsection{D data visualized in the National Geoportal}

The 3D datasets for piloting the 3D visualization included Helsinki city 3D buildings and Tampere city 3D buildings from the NTDB and Kuopio city point clouds produced by the NLS. The 3D building data to the NTDB were produced by the cities of Helsinki and Tampere since the NLS 3D building production process hasn't started yet. 3D building datasets from the NTDB were processed through the 3D service pipeline. Because the database doesn't support textures yet, the styles were set manually.

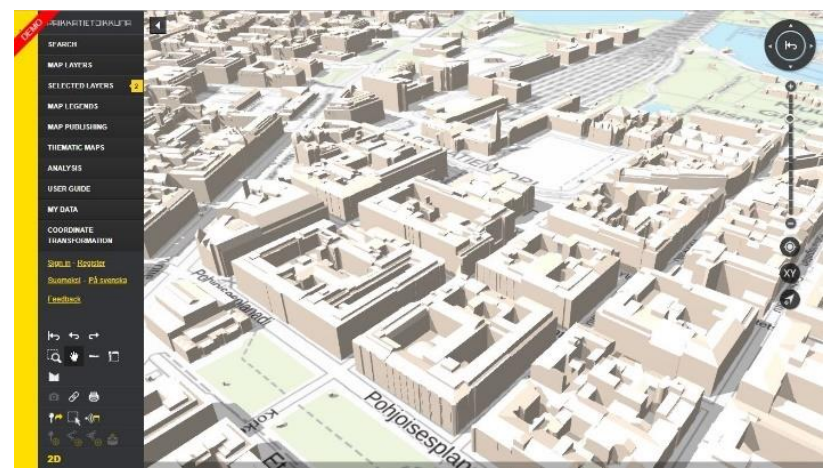

Figure 5 Helsinki 3D buildings from the NTDB

Some of the Tampere city buildings had also property information and those buildings are visualized with red color. The information can be seen by clicking the building.

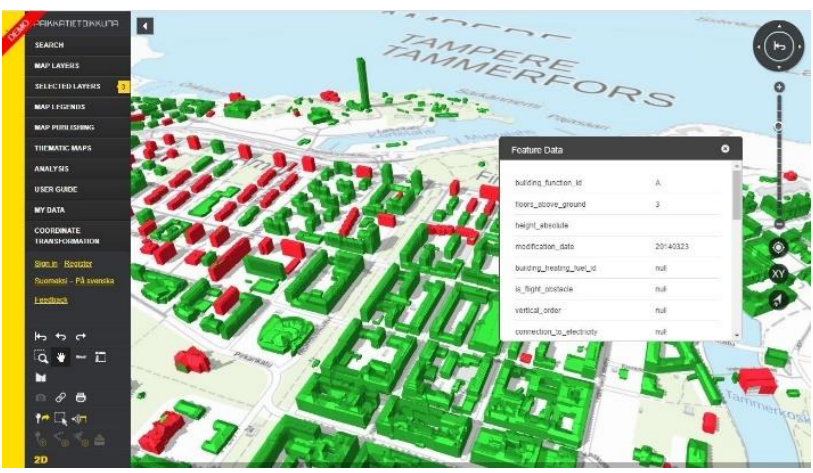

Figure 6 Tampere city 3D buildings with property information

Helsinki city 3D model by the Helsinki Region Infoshare (HRI) was also used for piloting the visualization to compare if the Cesium ion 3D tiling was as successful for the data from different sources. The data of HRI was already in the CityGML format and it was uploaded straight to Cesium ion. The HRI data had some errors that had to be corrected before it could be successfully imported to Cesium ion.

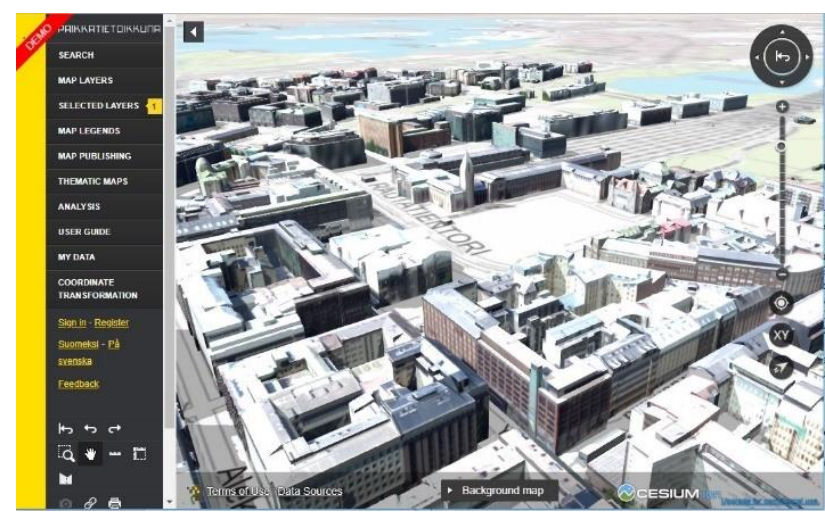

Figure 7 HRI 3D buildings in the National Geoportal

Cesium ion supports also point cloud data. Kuopio city point cloud data was imported to Cesium ion and visualized in the National Geoportal by configuring the size and colors of the points.
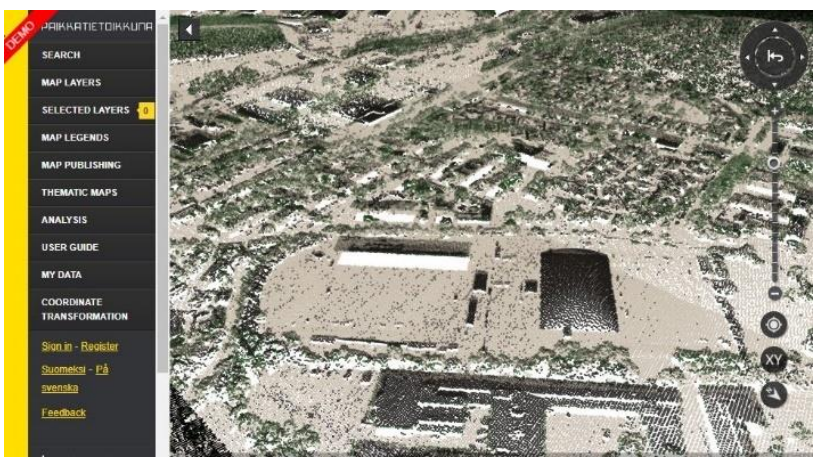

Figure 8 Kuopio city point cloud data

\section{DISCUSSION}

The current iteration of 3D buildings is focused on creating the initial country-wide 3D building repository. As the next 6-year cycle approaches, the updating process of the 3D buildings must be investigated. As the production is currently based on the laser scanning data, it is possible that a building's point cloud data might have been higher quality on the previous scanning round than the newer one. This requires a process where we compare the old 3D buildings to the new 3D buildings and determine which ones should be the primary data in the repository. The updating process also creates challenges for the $3 \mathrm{D}$ tile production. It is still unclear if the tiles in our workflow can be indexed such that only the tiles that have changed need to be updated or if the whole 3D tile dataset must be re-created.

Building 3D SDI for the whole country is a massive task that requires co-operation between different parts of the project. The support for $3 \mathrm{D}$ visualization was developed at the same time with the 3D data collection infrastructure and the 3D service. This enabled agile development not only inside the sub-projects but also between them, helping them to meet the requirements they set for each other. The drawback still was that only small amount of 3D data was available at the time of developing the support for 3D visualization. Therefore, the evaluation of the software performance was difficult. It was also hard to evaluate the size of the server capacity needed for the 3D service.

Currently, the use cases of the National 3D SDI of Finland are unclear. This project offers the basic 3D infrastructure as a starting point for companies and public sector to take the full 
advantage of the continuously developing 3D data. Identifying the needs for 3D information by potential users could be used for demonstrating the use cases in the National Geoportal. This could help other organizations to identify how they could use the 3D information. In the Netherlands there has been a study (Stoter et al., 2011) regarding the user needs for 3D SDI and some of these use cases could be demonstrated in the National Geoportal.

\section{CONCLUSIONS AND FUTURE WORK}

The Public Administration Recommendation that is currently under development creates a first step towards higher degree of harmonization between the data producers. The 3D modelling guidelines, which are a part of the recommendation, create a basis for the 3D data produced in Finland. The guidelines still require revising. As the experience with the guidelines grows in practice and the produced 3D data gets adopted by more and more users, the guidelines can be updated to function as an all-purpose modelling guide for 3D spatial data.

With this project, the framework for the 3D SDI has been developed, but it requires still efforts for getting the municipalities involved in the $3 \mathrm{D}$ data production. This can be promoted by communicating the benefits of the data harmonization. In addition, the guidance and specifications must be easily accessible and understandable.

The process of visualizing the 3D data from the NTDB was piloted in this project with only a few datasets. In the future development still needs to be done regarding every part of the process in order to handle the country-wide 3D spatial data. The performance of the visualization process is a key issue regarding the user experience. The updating process for the 3D data must also be developed in the future.

\section{REFERENCES}

Acil Allen Consulting and CRC for Spatial Information, 2017. Economic Value of Spatial Information in NSW.

Ahonen-Rainio, P., Mäkelä, J., Virrantaus, K. 2014. Menetelmä avoimen maastotiedon vaikuttavuuden arvioimiseksi. Tutkimusraportti. Aalto-yliopisto, Maankäyttötieteiden laitos, Geoinformatiikan tutkimusryhmä.

Biljecki, F., Ledoux, H., Stoter, J., Zhao, J., 2014. Formalisation of the level of detail in 3D city modelling, Environment and Urban Systems, 48, pp. 1-15.

Capstick, D., Heathcote, G., Horgan, J., \& Sargent, I. 2007. Moving towards 3D: from a National Mapping Agency perspective. The Cartographic Journal, 44(3), 233-238.

Eriksson, H., Harrie, L., \& Paasch, J. M. 2018. What is the need for building parts? - A comparison of CityGML, INSPIRE building and a Swedish building standard. International Archives of the Photogrammetry, Remote Sensing \& Spatial Information Sciences.

Finnish Ministry of Agriculture and Forestry, 2018. Report on spatial data policy. ISBN: 978-952-453-980-7 (PDF)

OGC, Open Geospatial Consortium, 2012. OGC City Geography Markup Language (CityGML) Encoding Standard. OGC document: 12-019
OGC, Open Geospatial Consortium, 2017. OGC Indexed 3d Scene Layer (I3S) and Scene Layer Package Format Specification. OGC document: 17-014r5

OGC, Open Geospatial Consortium, 2019. 3D Tiles Specification 1.0. OGC document: 18-053r2

Oude Elberink, S., Stoter, J., Ledoux, H., \& Commandeur, T. (2013). Generation of a National Virtual 3D City and Landscape Model for the Netherlands. Jaarverslag 2012 Nederlandse Commissie voor Geodesie, KNAW-NCG, 2013, pp. 41-56.

SIG 3D / Quality Working Group, 2013. Modeling guide for 3D objects - part 2: Modeling of buildings (LoD1, LoD2, LoD3)

Stoter, J., van den Brink, L., Vosselman, G., Goos, J., Zlatanova, S., Verbree, E., Klooster, R., van Berlo, L., Vestjens, G., Reuvers, M., Thorn, S. 2011. A generic approach for 3D SDI in the Netherlands. In Proceedings of the Joint ISPRS Workshop on 3D City Modelling\&Applications and the 6th 3D GeoInfo Conference Wuhan, China (pp. 26-28). 